\title{
Advanced neoplasia detection using chromoendoscopy and white light colonoscopy for surveillance in patients with inflammatory bowel disease
}

\author{
Kyeong Ok Kim ${ }^{1,2}$, Michael V. Chiorean ${ }^{1}$ \\ ${ }^{1}$ Digestive Disease Institute, Virginia Mason Medical Center, Seattle, WA, USA; ${ }^{2}$ Division of Gastroenterology and Hepatology, Department of \\ Internal Medicine, Yeungnam University College of Medicine, Daegu, Korea
}

Background/Aims: Chromoendoscopy (CE) has been shown to be superior to white light endoscopy (WLE) for neoplasia detection in inflammatory bowel disease (IBD). We aimed to compare the yield of CE and WLE for the detection of overall neoplasia and advanced neoplasia in IBD. Methods: Patients who underwent surveillance colonoscopy from 1999 to 2017 were identified from our IBD database. CE procedures were compared with their respective WLE controls in a paired comparison, and frequency of all neoplasia, advanced neoplasia, and serrated neoplasia was assessed for both targeted and random biopsies. Results: A total of 290 procedures performed in 98 individuals were identified with a median follow-up 4 years (median 3 colonoscopies/patient). CE and WLE were performed in 159 and 131 episodes, respectively. CE detected neoplasia in $40.9 \%$ of colonoscopies versus $23.7 \%$ with WLE $(P=0.002)$. In addition, CE detected more advanced neoplasia $(18.2 \%$ vs. $6.1 \%, P=0.002)$ and serrated lesions ( $14.5 \%$ vs. $6.1 \%, P=0.022)$. Significantly fewer samples were obtained per procedure with CE ( $14.9 \pm 9.7$ vs. $20.9 \pm 11.1, P<0.001)$. Cancer was diagnosed in 2 cases. Conclusions: CE has a higher detection rate than WLE for advanced neoplasia and serrated lesions in patients with IBD under surveillance. Further prospective studies evaluating the impact of CE on decreasing the risk of interval cancer and colectomy in IBD patients are warranted. (Intest Res 2020;18:438-446)

Key Words: Image enhancement; Colorectal neoplasms; Inflammatory bowel disease

\section{INTRODUCTION}

Inflammatory bowel diseases (IBD) including ulcerative colitis (UC) or Crohn's disease (CD) are chronic relapsing immunemediated conditions of the gastrointestinal tract requiring lifelong treatment. ${ }^{1}$ It is well known that long standing colitis can increase the risk of colon cancer, along with other risk factors such as histologically severe inflammation, disease extent, family history of colorectal cancer (CRC), and coexistent primary sclerosing cholangitis (PSC). ${ }^{2-4}$ As with sporadic colon

Received July 26, 2019. Revised December 15, 2019. Accepted April 29, 2020. Correspondence to Michael V. Chiorean, Digestive Disease Institute, Virginia Mason Medical Center, 1100 Ninth Ave, C3-GAS, Seattle, WA 98101, USA. Tel: +1-206-625-7373, Fax: +1-206-341-1405, E-mail: Michael.Chiorean@ virginiamason.org cancer, dysplasia is considered to be the precursor of most IBDrelated CRC. ${ }^{5}$ Based on this evidence, most guidelines recommend surveillance colonoscopy for the detection and treatment of dysplastic lesions with the goal of decreasing the morbidity and mortality from CRC. ${ }^{6-9}$

Although most dysplasia in IBD is associated with endoscopically visible mucosal lesions, neoplastic lesions are often difficult to detect due to the flat morphology and surrounding chronic inflammation. ${ }^{10-12}$ In an attempt to circumvent this issue, previous guidelines recommended obtaining random biopsies throughout the colon in order to increase the detection of "invisible dysplasia." The yield of random biopsies is extremely low, the cost associated with the large number of tissue samples is high, and this strategy has not been shown to decrease the incidence of colon cancer. ${ }^{13}$ Dye chromoendoscopy (CE), 
by using indigo carmine or methylene blue, is a promising technique used to increase the detection of neoplastic mucosal lesion by identifying subtle changes in the surface epithelium or pit pattern. Several randomized clinical trials and case control studies have shown that CE can detect significantly more neoplasia compared with white light endoscopy (WLE) with or without random biopsies. ${ }^{14-17}$ In addition, a recent study showed that CE is more cost-effective than WLE for UC surveillance, regardless of the surveillance interval. ${ }^{18}$ Based on these results, major society guidelines recommend using CE with targeted biopsies as the modality of choice for surveillance in IBD patients. ${ }^{6-8}$ There are, however, several limitations to CE such as a long procedure time, additional cost for the dye and equipment, and the lack of systematic endoscopic training. ${ }^{19}$ Given the lack of long-term outcomes with CE, several studies have raised doubts about the significance of the additional dysplasia detected by CE as most of these lesions may have low risk of progression to advanced neoplasia or cancer. In addition, recent studies have questioned the superiority of CE over high-definition WLE in detection neoplasia in patients with chronic colitis. ${ }^{20}$

Compared with sporadic colon cancer, the transition from inflamed epithelium to low-grade dysplasia (LGD), high-grade dysplasia (HGD) and eventually invasive carcinoma may occur faster in patients with IBD. Thus, detecting advanced neoplastic lesions during surveillance in these patients may be a more relevant endpoint for decreasing the risk of CRC. Serrated lesions (sessile serrated polyps/adenoma and traditional serrated adenomas) have also been associated with an increased risk of metachronous multifocal dysplasia in patients with IBD thus being indirectly implicated in the pathogenesis of colitis associated CRC. ${ }^{21-23}$ Whether CE has an increased detection ability for serrated lesions compared with WLE is unknown.

In this study, we aimed to compare the yield of CE and WLE for the detection of all neoplasia, advanced neoplasia, and serrated lesions and assess the utility of random versus targeted biopsies for the surveillance of neoplasia in patients with IBD.

\section{METHODS}

\section{Patients}

From January 1999 to August 2017, patients with IBD, including both UC/CD and IBD undifferentiated (IBDU), who underwent surveillance colonoscopy based on existing guidelines (i.e., extensive colitis $>8$-year duration, left-sided colitis $>15$-year duration, previous history of dysplasia or coexistent PSC) at the Virginia Mason Medical Center were retrospectively identified using the institution-based IBD database repository. In order to be eligible, patients had to have at least 1 surveillance colonoscopy using a high-definition colonoscope, have more than proctitis or over $30 \%$ colon involvement for CD. Patients with prior total or hemi colectomy and a history of CRC were excluded. Patients with CD who underwent ileocecectomy were included in the analysis. The diagnosis of UC and $\mathrm{CD}$ was based on clinical and endoscopic criteria included in the most recent guidelines. ${ }^{24,25}$ The index colonoscopy was defined as the 1st surveillance colonoscopy performed at this institution and procedures may have been performed using either WLE or CE at the discretion of the provider. Index colonoscopies performed at the time of diagnosis were excluded. Patients' baseline demographics and disease characteristics, prior history of dysplasia or PSC, type of surveillance procedure (standard vs. high-definition, white light vs. CE) and the endoscopic, pathological and surgical diagnoses were collected using the electronic medical records which encompasses medical information dating back to 1996.

After 2008, all colonoscopies at our institution were performed using high-definition endoscopes (CF-H180AL/I and PCFH180AL/I; Olympus, Tokyo, Japan). CE was performed after 2012 using $0.2 \%$ indigo carmine in the vast majority of cases, or $0.1 \%$ methylene blue. The dye agent was applied either directly using the endoscopic flushing pump (OFP-2; Olympus America, Center Valley, PA, USA) or by spray catheter (Olympus PW-205V; Olympus Europe, Hamburg, Germany) upon withdrawal of the scope, and both targeted and random biopsies were performed at the discretion of the endoscopist. All CE were performed by a single experienced endoscopist with more than 10 years of experience with this technique. All WLE procedures were performed by gastroenterologists with at least 5 years of experience after completing training. Patients who underwent both CE and WLE served as their own controls. The withdrawal time was calculated by the time difference between the last anal and 1st cecal image after terminal ileal intubation.

The number of visible lesions, targeted and random biopsies, and pathological diagnosis were collected from the endoscopic mucosal resection (EMR). The lesion morphology was described based on the SCENIC guidelines in polypoid, nonpolypoid, and invisible. ${ }^{7}$ Advanced neoplastic lesions were defined as larger than $10 \mathrm{~mm}$ in diameter, harboring HGD or villous features. The histopathology was categorized according 
to the Riddle classification in negative, indefinite, LGD or HGD and invasive carcinoma. ${ }^{26}$ Serrated lesions including sessile serrated polyps and traditional serrated adenomas were diagnosed based on the crypt architecture by pathologists with extensive experience in gastroenterology. The number of samples collected during each procedure was estimated by the summation of the number of tissue samples found in each jar as described in the pathology report. Samples from discrete lesions such as polyps or non-polypoid lesions were considered as one unit whereas random biopsies were counted independently. The bowel preparation status was assessed by both Aronchick and Boston Bowel Preparation Scale with scores such as fair, good, and excellent being considered acceptable. The study was approved by the Benaroya Research Institute Institutional Review Board (IRB No. IRB17-110) and written informed consent by the patients was waived due to a retrospective nature of our study.

\section{Statistical Analysis}

The incidence and prevalence of neoplasia were calculated using standard methodology and averaged based on the length of follow-up. Per procedure comparisons of the yield for overall neoplasia, advanced neoplasia and serrated pathology between CE and WLE were performed by accounting each procedure as an individual "event or episode" thus allowing comparisons both within and between patients. Patients' baseline demographics and disease characteristics, number of targeted and random biopsies were also compared between groups using standard statistical methods.

Continuous variables were expressed as median or mean \pm standard deviation and compared using the 2 independent samples median test for median or Student $t$-test for mean value. Categorical variables were compared using Pearson chisquare analysis or Fisher exact test. Statistical significance was defined as a $P$-value of less than 0.05 . All statistical analyses were performed using the Statistical Package for Social Sciences, IBM SPSS version 23.0 (IBM Corp., Armonk, NY, USA).

\section{RESULTS}

A total of 106 patients underwent 315 surveillance colonoscopies (median 3/patient; range, 1-13) during a median followup period of 4 years (range, 0.5-12.5 years) for a total of 914 patient-years of follow-up. The median surveillance interval between colonoscopies was 15 months (range, 4-98 months). Eight patients were excluded based on disease limited to the rectum $(\mathrm{n}=1)$, CD with exclusive small bowel involvement $(\mathrm{n}=3)$, prior total colectomy $(\mathrm{n}=2)$, and prior history of colon cancer $(n=2)$. A total of 290 surveillance colonoscopies from 98 patients were included in the final analysis, of which 7 procedures $(2.4 \%)$ were performed using standard definition colonoscopes prior to 2008. Twenty-four patients had CD (24.5\%), 68 UC (69.4\%), and the other 6 had IBDU. The median disease duration was 16.5 years (range, 1-60 years) and median age at diagnosis was 30 years (range, 11-75 years). Among UC patients, 48 had extensive colitis (E3, 70.6\%) and 20 left-sided colitis (E2, 29.4\%). Coexistent PSC was present in $11.2 \%$ of patients.

Of the total of 290 procedures (episodes), 159 were performed using CE (54.8\%). Demographic and baseline characteristics of the entire cohort are shown in Table 1. Family history of CRC was more prevalent in the WLE group, but active smoking and PSC were more common in the CE group. Follow-up duration and extent were not significantly different between the 2 groups. In $52.7 \%$ of procedures (150/290), the disease was in endoscopic remission (61.0\% in CE and $40.5 \%$ in WLE)

Table 1. Baseline Characteristics of the Patients Included in the Study

\begin{tabular}{lccc}
\hline Characteristics & $\begin{array}{c}\text { Chromo- } \\
\text { endoscopy } \\
(\mathrm{n}=159)\end{array}$ & $\begin{array}{c}\text { White light } \\
\text { endoscopy } \\
(\mathrm{n}=131)\end{array}$ & P-value \\
\hline Age (yr) & $59(20-81)$ & $57(26-87)$ & 0.900 \\
Male sex & $92(57.9)$ & $64(48.9)$ & 0.160 \\
Smoking & $9(5.7)$ & $5(3.8)$ & 0.044 \\
IBD diagnosis & $110(69.2)$ & $72(55.0)$ & 0.028 \\
UC & $42(26.4)$ & $54(41.2)$ & \\
Crohn's colitis & $7(4.4)$ & $5(3.8)$ & \\
IBDU & $31(11-75)$ & $27(13-71)$ & 0.605 \\
Age at diagnosis (yr) & $16(1-60)$ & $15(1-59)$ & 0.945 \\
Follow-up duration (yr) & & & \\
Disease extent & $75(47.2)$ & $48(36.6)$ & 0.940 \\
Extensive UC & $35(22.0)$ & $24(18.3)$ & \\
Left sided UC & $35(22.0)$ & $37(28.2)$ & 0.150 \\
Multisegment CD & $7(4.4)$ & $17(13.0)$ & \\
Segmental CD & $22(13.8)$ & $32(24.4)$ & 0.047 \\
Family history of CRC & $16(10.1)$ & $5(3.8)$ & 0.041 \\
\hline PSC diagnosis & & & \\
\hline
\end{tabular}

Values are presented as median (range) or number (\%). IBD, inflammatory bowel disease; UC, ulcerative colitis; IBDU, IBD undifferentiated; $C D$, Crohn's disease; $C R C$, colorectal cancer; PSC, primary sclerosing cholangitis. 
Table 2. Classification of Findings at Chromoendoscopy and White Light Endoscopy by Morphological and Histological Criteria

\begin{tabular}{llccc}
\hline Morphology & Histology & $\begin{array}{c}\text { Chromo- } \\
\text { endoscopy }\end{array}$ & $\begin{array}{c}\text { White light } \\
\text { endoscopy }\end{array}$ & P-value \\
\hline Polypoid & LGD & $37(40.2)$ & $19(32.6)$ & 0.040 \\
& HGD & $3(3.3)$ & 0 & 0.080 \\
& SSL & $11(12.0)$ & $6(14.0)$ & 0.120 \\
& IND & $1(1.1)$ & $1(2.3)$ & 0.820 \\
& Cancer & $1(1.1)$ & 0 & 0.360 \\
& Total & $53(57.6)^{\mathrm{a}}$ & $26(60.5)^{\mathrm{a}}$ & 0.020 \\
Non-polypoid & LGD & $21(22.8)$ & $13(30.2)$ & 0.360 \\
& HGD & $3(3.3)$ & $1(2.3)$ & 0.400 \\
& SSL & $14(15.2)$ & $3(7.0)$ & 0.012 \\
& IND & $1(1.1)$ & 0 & 0.360 \\
& Total & $39(42.4)^{\mathrm{a}}$ & $17(39.5)^{\mathrm{a}}$ & 0.030 \\
\hline Advanced & & $41(44.6)$ & $7(16.3)$ & $<0.001$ \\
neoplasia & & & & \\
\hline
\end{tabular}

Values are presented as number (\%).

${ }^{a}$ Proportions of overall polypoid and non-polypoid lesions for each method. LGD, low-grade dysplasia; HGD, high-grade dysplasia; SSL, sessile serrated lesions including both sessile serrated polyps and traditional serrated adenoma; IND, indefinite for dysplasia. in $42.1 \%$ (122/290), there was mild or moderate activity (37.7 in CE and $47.3 \%$ in WLE) and in the remaining 18 procedures (5.2\%) there was severe inflammation (1.3\% in CE and $12.2 \%$ in WLE). The bowel preparation status was acceptable in $95.6 \%$ of CE and $91.6 \%$ of WLE procedure $(P=0.11)$. Withdrawal time was significantly longer in CE $(25.0 \pm 9.6$ minutes vs. $18.9 \pm 6.7$ minutes, $P<0.001)$. EMR was performed in $29.6 \%$ of CE episodes versus $9.2 \%$ in the WLE group $(P<0.001)$. There were no serious adverse events during either CE or WLE including EMR cases.

\section{Diagnostic Yield}

The most common morphological type of neoplasia was polypoid (57.6\% and $60.5 \%$, respectively) in both groups. CE detected both more polypoid and non-polypoid lesions compared with WLE. Considering both morphological and histological criteria, CE detected more polypoid LGD and more non-polypoid serrated lesions. The overall neoplasia detection rate per procedure was $40.9 \%(65 / 159)$ in the CE group and $23.7 \%(31 / 131)$ in WLE group $(P=0.002)$. CE also detected overall significantly more advanced neoplastic lesions compared with WLE $(P<0.001)$ as shown in Table 2.

Table 3. Per Procedure Neoplasia Detection Rate by Chromoendoscopy and White Light Endoscopy

\begin{tabular}{lccc}
\hline Outcome & Chromoendoscopy $(n=159)$ & White light endoscopy $(n=131)$ & $P$-value \\
\hline Neoplasia per episode & $65(40.9)$ & $31(23.7)$ & 0.002 \\
Advanced neoplasia per episode & $29(18.2)$ & $8(6.1)$ & 0.002 \\
Serrated lesion per episode & $23(14.5)$ & $8(6.1)$ & 0.022 \\
Targeted biopsies & $1.3 \pm 1.2$ & $0.7 \pm 1.0$ & $<0.001$ \\
Neoplasia per targeted biopsy (\%) & 42.5 & 42.7 & 0.820 \\
LGD & 26.3 & 33.2 & 0.250 \\
HGD & 1.9 & 0 & 0.033 \\
Serrated & 13.0 & 9.2 & 0.420 \\
Indefinite & 0.4 & 0.3 & 0.880 \\
Cancer & 0.9 & 0 & 0.510 \\
Advanced neoplasia (\%) & 17.3 & 6.1 & 0.034 \\
Random biopsy & $13.56 \pm 9.34$ & $20.23 \pm 10.64$ & $<0.001$ \\
Neoplasia per random biopsy (\%) & 0.19 & 0.19 & 0.490 \\
LGD & 0.14 & 0.11 & 0.460 \\
HGD & 0 & 0.04 & 0.120 \\
Serrated & 0 & 0.04 & 0.800 \\
Indefinite & 0.05 & 0 & 0.510 \\
\hline
\end{tabular}

Values are presented as number (\%) or mean \pm standard deviation.

LGD, low-grade dysplasia; HGD, high-grade dysplasia. 
The mean total number of samples and the mean number of random biopsies per procedure were lower in CE versus WLE $(14.9 \pm 9.7$ vs. $20.9 \pm 11.1, P<0.001$ [data not shown] and $13.6 \pm 9.3$ vs. $20.2 \pm 10.6, P<0.001)$. However, significantly more targeted biopsies were performed during CE compared with WLE $(1.3 \pm 1.2$ vs. $0.7 \pm 1.0, P<0.001)$. The proportion of neoplastic lesions per targeted biopsy was not significantly different between CE and WLE ( $42.5 \%$ vs. $42.7 \%, P=0.82$ ), but there was a higher proportion of advanced neoplasia per targeted biopsy in the CE group (Table 3). The proportion of random biopsies positive for dysplasia was $0.19 \%$ in both groups $(P=0.49)$, with a number needed to test of 526 for both CE and WLE to detect one invisible dysplasia (Table 3). The differences between CE and WLE remained robust for all outcomes even after excluding the 7 procedures $(2.4 \%)$ performed with standard definition scopes prior to 2008. The overall prevalence of neoplasia in this cohort was $48.0 \%$, with an average incidence rate of 0.49 per year. Among the patients with incidental LGD detected during surveillance $(\mathrm{n}=18), 12$ patients (66\%) developed metachronous LGD, 1 patient HGD (5.5\%),

Table 4. Surveillance Outcomes of Patients with Neoplasia Resection at Baseline Colonoscopy

\begin{tabular}{|c|c|c|c|c|c|c|}
\hline Index & Histology & $\begin{array}{c}1 \text { st } \\
\text { surveillance }\end{array}$ & $\begin{array}{c}\text { 2nd } \\
\text { surveillance }\end{array}$ & $\begin{array}{c}\text { 3rd } \\
\text { surveillance }\end{array}$ & $\begin{array}{c}\text { 4th } \\
\text { surveillance }\end{array}$ & $\begin{array}{c}\text { 5th surveillance } \\
\text { or more }\end{array}$ \\
\hline \multirow[t]{4}{*}{ LGD alone $(n=18)$} & Negative & 10 & 9 & 4 & 0 & 1 \\
\hline & LGD & 6 & 4 & 3 & 4 & 6 \\
\hline & $\mathrm{HGD}$ & 0 & 1 & 1 & 0 & 0 \\
\hline & Cancer & 0 & 1 & 0 & 0 & 0 \\
\hline \multirow[t]{3}{*}{ LGD and SSL $(n=5)$} & Negative & 0 & 1 & NA & NA & NA \\
\hline & LGD & 2 & 0 & - & - & - \\
\hline & HGD & 0 & 0 & - & - & - \\
\hline \multirow[t]{3}{*}{ HGD alone $(n=2)$} & Negative & 0 & NA & NA & NA & NA \\
\hline & LGD & 0 & - & - & - & - \\
\hline & HGD & 1 & - & - & - & - \\
\hline \multirow[t]{3}{*}{ SSL alone $(n=8)$} & Negative & 4 & 2 & NA & NA & NA \\
\hline & LGD & 0 & 0 & - & - & - \\
\hline & HGD & 0 & 0 & - & - & - \\
\hline
\end{tabular}

Data are presented as number. There were no patients with coexistent HGD and SSL at baseline.

LGD, low-grade dysplasia; HGD, high-grade dysplasia; SSL, sessile serrated lesion; NA, not available.

Table 5. Clinicopathological Findings of Colectomy Cases

\begin{tabular}{|c|c|c|c|c|c|c|}
\hline Patient No. & Diagnosis & $\begin{array}{c}\text { Surveillance } \\
\text { method }\end{array}$ & $\begin{array}{l}\text { Endoscopy } \\
\text { result }\end{array}$ & $\begin{array}{c}\text { Lesion by random } \\
\text { biopsy }\end{array}$ & $\begin{array}{l}\text { Colectomy } \\
\text { findings }\end{array}$ & $\begin{array}{c}\text { Colonoscopy } \\
\text { correlation }\end{array}$ \\
\hline 1 & UC & $\mathrm{CE}$ & $H G D^{a}$ & No & HGD & Yes \\
\hline 2 & UC & WLE & NR HGD & Yes & Cancer & $\mathrm{No}^{\mathrm{b}}$ \\
\hline 3 & UC & $\mathrm{CE}$ & NR HGD & No & $\mathrm{HGD}$ & Yes \\
\hline 4 & UC & $\mathrm{CE}$ & NR LGD & No & LGD & Yes \\
\hline 5 & UC & $\mathrm{CE}$ & NR TSA & No & TSA & Yes \\
\hline 6 & $C D$ & CE & NR LGD & No & LGD & Yes \\
\hline
\end{tabular}

Incompletely resected.

${ }^{6}$ The cancer detected after colectomy was remote from the HGD lesion. In endoscopy, there was an area with multiple pseudopolyps at left side colon. The tissue was taken randomly at the site, but the biopsy showed just chronic inflammation. Because there was another NR HGD at right side colon, total colectomy was performed and the cancer at left side colon was diagnosed.

UC, ulcerative colitis; CD, Crohn's disease; CE, chromoendoscopy; WLE, white light endoscopy; HGD, high-grade dysplasia; NR, non-resectable; LGD, lowgrade dysplasia; TSA, traditional serrated adenoma. 
and 1 developed adenocarcinoma during follow-up (Table 4).

\section{Histologic Findings after Colectomy}

Colectomy was performed in 6 patients (5.7\%), and 5 of them had a prior CE. One patient had an incompletely resected HGD lesion, and the other cases were non-resectable lesions (2 HGD and 3 LGD). In all but 1 case there was perfect correlation between the endoscopic and surgical diagnosis (Table 5). Of the 2 cancer cases, 1 was detected by CE, and the other was previously diagnosed as HGD by WLE, 2 months before colectomy. One patient with cancer had LGD diagnosed on 2 consecutive surveillance colonoscopies prior to the cancer diagnosis, and the other patient had no prior diagnosis of dysplasia. One patient refused surgery and died of metastatic disease 2.5 years later.

\section{DISCUSSION}

In this study, we have shown that CE has a higher diagnostic yield for neoplasia and particularly advanced neoplasia, compared with high-definition WLE in patients with IBD. Per procedure, neoplasia detection rate was nearly double, and there were almost three times as many advanced neoplastic lesions detected by CE compared with WLE. Based on the available evidence regarding the superior yield for neoplasia, CE was recommended as the modality of choice for IBD surveillance by several major medical societies. ${ }^{6-8}$ However, there are barriers to the implementation of CE in clinical practice. On a recent survey, only $30 \%$ of IBD specialists routinely use CE. ${ }^{27}$ One of the reasons for the slow uptake of CE has been the lack of long-term data supporting an association between a higher neoplasia detection rate and a lower risk of cancer. While there is controversy as to the impact of non-advanced lesions detected by $\mathrm{CE}$, the data seems more meaningful in regard to the advanced lesions (larger size or containing HGD). Choi et al. ${ }^{28}$ showed that the rate of interval cancers was reduced in half following a negative CE compared with a normal WLE. The prevalence of dysplasia in our study (48.0\%) was higher compared with other cohorts, but the incidence was similar. ${ }^{28,29}$ This may be due to referral bias, a higher proportion of patients with major risk factors such as PSC, or the systematic use of high-definition endoscopy. In our institution, we also employed pan-chromoendoscopy as opposed to targeted dye spraying as was the case in some studies.

Most neoplastic lesions in our study were polypoid, which is consistent with previous publications. ${ }^{14,30-33}$ Rutter et al. ${ }^{31}$ and
Rubin et $\mathrm{al} .^{32}$ have shown that most neoplasia in patients with IBD is visible. In our study, CE detected significantly more elevated lesions leading to more targeted biopsies compared with WLE (213 vs. 89). Overall, our data confirms that CE has a higher sensitivity for neoplastic lesions compared with WLE. We have to acknowledge that our results are discordant to findings from other recent studies showing no superiority of CE over WLE. ${ }^{20,34,35}$ However, these studies excluded patients at high-risk for neoplasia such as those with coexistent PSC which, again, may have biased the results.

An important finding from our study is the higher yield for advanced neoplasia. CE detected almost three times as many advanced neoplastic lesions compared with WLE, and per lesion advanced neoplasia yield was also significantly higher, although high-definition scopes were used in the vast majority of cases. The difference between the 2 methods remained robust even after excluding the 7 procedures performed with standard definition WLE colonoscopy prior to 2008. This is a very important finding again supporting the use of CE over WLE for IBD surveillance. The decreased incidence of interval cancer in the study of Choi et al. ${ }^{28}$ may also be due in part to a higher detection rate for advanced neoplasia, although this was not specifically addressed.

The detection rate for serrated lesions was also higher with CE compared with WLE in this study. CE has been shown to have a higher detection rate for serrated lesions in patients without IBD. ${ }^{36,37}$ Recent reports have suggested that IBD patients with serrated neoplasia at baseline have a higher risk of developing conventional dysplasia including advanced neoplasia in follow-up. ${ }^{21-23}$ Therefore, detecting these often inconspicuous lesions may have an important impact in reducing the risk of interval cancers in IBD patients.

There were significantly more targeted biopsies and fewer random biopsies obtained during CE compared with WLE. Similar to previous studies, the yield of random biopsies for dysplasia was extremely low. ${ }^{14,16,38}$ Thus, CE may represent a cost-saving method for IBD surveillance without compromising diagnostic accuracy. This is consistent with data from Konijeti et al. ${ }^{18}$ showing that CE may be more cost-effective for IBD surveillance regardless of the surveillance interval.

Our study has several limitations resulting from its retrospective nature and the referral bias which likely led to the inclusion of patients with a higher risk for neoplasia compared with population-based studies. There was a slight imbalance between groups, particularly in regard to the distribution of patients with PSC and family history of colon cancer. In our ret- 
rospective review of medical and procedural records, we may have omitted other potential confounders that could have increased the imbalance between the groups and spuriously augmented the yield of CE over WCE. Such biases can only be mitigated in prospective randomized trials. All CEs were performed by a single experienced endoscopist, and it is unclear if this can be replicated in other clinical settings. However, the CE learning curve may not be steep; in a recent study, the yield of dysplasia during CE was not different between expert and novice endoscopists following a short training course. ${ }^{39} \mathrm{We}$ could not make a distinction between colitis-associated and sporadic neoplasms as most patients were in endoscopic remission and the histological disease extent was not adequately documented in all cases. However, our aim was to compare the yield of CE with WLE for IBD surveillance regardless of the disease extent and there is no reason to suspect that the inclusion of sporadic lesions has introduced a bias in favor of CE.

The relatively short follow-up duration and small number of patients also limit the generalizability of our results. Chronologically, CE procedures were performed during more recent years, so a calendar-year bias cannot be excluded. In addition, 7 exams before 2008 in WLE group were performed by standard definition. Although the proportion was relative low, it could influence on the result. All pathological specimens were internally reviewed, and observer bias may be a consideration, although this should be relatively balanced between CE and WLE.

In conclusion, we demonstrated that CE has a higher detection rate for all neoplasia and particularly advanced and serrated neoplasia compared with white light, high-definition colonoscopy. The yield of random biopsies performed during either CE or WLE was extremely low, which raises serious doubts about the utility of this method for neoplasia surveillance. Although further prospective studies are necessary, CE seems to be a useful technique for neoplasia surveillance in patients with IBD.

\section{FINANCIAL SUPPORT}

The authors received no financial support for the research, authorship, and/or publication of this article.

\section{CONFLICT OF INTEREST}

No potential conflict of interest relevant to this article was reported.

\section{AUTHOR CONTRIBUTION}

Conceptualization: Chiorean MV. Methodology: Chiorean MV, Kim KO. Formal analysis: Chiorean MV, Kim KO. Project administration: Chiorean MV. Visualization: Chiorean MV, Kim KO. Writing original article: Kim KO. Writing-review and edition: Chiorean MV, Kim KO. Approval of final manuscript: all authors.

\section{ORCID}

Kim KO

Chiorean MV https://orcid.org/0000-0001-5799-7436 https://orcid.org/0000-0002-4238-9988

\section{REFERENCES}

1. Kim ES. Role of advanced endoscopic imaging techniques in the management of inflammatory bowel disease. Clin Endosc 2017;50:424-428

2. Ekbom A, Helmick C, Zack M, Adami HO. Ulcerative colitis and colorectal cancer: a population-based study. N Engl J Med 1990;323:1228-1233.

3. Ekbom A, Helmick C, Zack M, Adami HO. Increased risk of large-bowel cancer in Crohn's disease with colonic involvement. Lancet 1990;336:357-359.

4. Colman RJ, Rubin DT. Histological inflammation increases the risk of colorectal neoplasia in ulcerative colitis: a systematic review. Intest Res 2016;14:202-210.

5. Thomas T, Abrams KA, Robinson RJ, Mayberry JF. Meta-analysis: cancer risk of low-grade dysplasia in chronic ulcerative colitis. Aliment Pharmacol Ther 2007;25:657-668.

6. Farraye FA, Odze RD, Eaden J, et al. AGA medical position statement on the diagnosis and management of colorectal neoplasia in inflammatory bowel disease. Gastroenterology 2010; 138:738-745.

7. Laine L, Kaltenbach T, Barkun A, et al. SCENIC international consensus statement on surveillance and management of dysplasia in inflammatory bowel disease. Gastrointest Endosc 2015;81:489-501.

8. Cairns SR, Scholefield JH, Steele RJ, et al. Guidelines for colorectal cancer screening and surveillance in moderate and high risk groups (update from 2002). Gut 2010;59:666-689.

9. Magro F, Gionchetti P, Eliakim R, et al. Third European evidencebased consensus on diagnosis and management of ulcerative colitis. Part 1: definitions, diagnosis, extra-intestinal manifestations, pregnancy, cancer surveillance, surgery, and ileo-anal 
pouch disorders. J Crohns Colitis 2017;11:649-670.

10. Shanahan F, Quera R. CON: surveillance for ulcerative colitisassociated cancer: time to change the endoscopy and the microscopy. Am J Gastroenterol 2004;99:1633-1636.

11. Fujii S, Fujimori T, Chiba T, Terano A. Efficacy of surveillance and molecular markers for detection of ulcerative colitis-associated colorectal neoplasia. J Gastroenterol 2003;38:11171125.

12. Itzkowitz SH, Harpaz N. Diagnosis and management of dysplasia in patients with inflammatory bowel diseases. Gastroenterology 2004;126:1634-1648.

13. van den Broek FJ, Stokkers PC, Reitsma JB, et al. Random biopsies taken during colonoscopic surveillance of patients with longstanding ulcerative colitis: low yield and absence of clinical consequences. Am J Gastroenterol 2014;109:715-722.

14. Rutter MD, Saunders BP, Schofield G, Forbes A, Price AB, Talbot IC. Pancolonic indigo carmine dye spraying for the detection of dysplasia in ulcerative colitis. Gut 2004;53:256-260.

15. Kiesslich R, Fritsch J, Holtmann M, et al. Methylene blue-aided chromoendoscopy for the detection of intraepithelial neoplasia and colon cancer in ulcerative colitis. Gastroenterology 2003;124:880-888.

16. Marion JF, Waye JD, Present DH, et al. Chromoendoscopy-targeted biopsies are superior to standard colonoscopic surveillance for detecting dysplasia in inflammatory bowel disease patients: a prospective endoscopic trial. Am J Gastroenterol 2008;103:2342-2349.

17. Kiesslich R, Goetz M, Lammersdorf K, et al. Chromoscopyguided endomicroscopy increases the diagnostic yield of intraepithelial neoplasia in ulcerative colitis. Gastroenterology 2007;132:874-882.

18. Konijeti GG, Shrime MG, Ananthakrishnan AN, Chan AT. Costeffectiveness analysis of chromoendoscopy for colorectal cancer surveillance in patients with ulcerative colitis. Gastrointest Endosc 2014;79:455-465.

19. ASGE Technology Committee, Wong Kee Song LM, Adler DG, et al. Chromoendoscopy. Gastrointest Endosc 2007;66:639649.

20. Mooiweer E, van der Meulen-de Jong AE, Ponsioen CY, et al. Chromoendoscopy for surveillance in inflammatory bowel disease does not increase neoplasia detection compared with conventional colonoscopy with random biopsies: results from a large retrospective study. Am J Gastroenterol 2015;110:10141021.

21. Jackson WE, Achkar JP, Macaron C, et al. The significance of sessile serrated polyps in inflammatory bowel disease. Inflamm
Bowel Dis 2016;22:2213-2220.

22. Polydorides AD, Harpaz N. Serrated lesions in inflammatory bowel disease. Gastrointest Endosc 2017;85:461.

23. Shen J, Gibson JA, Schulte S, et al. Clinical, pathologic, and outcome study of hyperplastic and sessile serrated polyps in inflammatory bowel disease. Hum Pathol 2015;46:1548-1556.

24. Dignass A, Eliakim R, Magro F, et al. Second European evidence-based consensus on the diagnosis and management of ulcerative colitis part 1: definitions and diagnosis. J Crohns Colitis 2012;6:965-990.

25. Dignass A, Van Assche G, Lindsay JO, et al. The second European evidence-based consensus on the diagnosis and management of Crohn's disease: current management. J Crohns Colitis 2010;4:28-62.

26. Riddell RH, Goldman H, Ransohoff DF, et al. Dysplasia in inflammatory bowel disease: standardized classification with provisional clinical applications. Hum Pathol 1983;14:931-968.

27. Gallinger ZR, Rumman A, Murthy SK, Nguyen GC. Perspectives on endoscopic surveillance of dysplasia in inflammatory bowel disease: a survey of academic gastroenterologists. Endosc Int Open 2017;5:E974-E979.

28. Choi CH, Rutter MD, Askari A, et al. Forty-year analysis of colonoscopic surveillance program for neoplasia in ulcerative colitis: an updated overview. Am J Gastroenterol 2015;110:10221034.

29. Marion JF, Waye JD, Israel Y, et al. Chromoendoscopy is more effective than standard colonoscopy in detecting dysplasia during long-term surveillance of patients with colitis. Clin Gastroenterol Hepatol 2016;14:713-719.

30. Hlavaty T, Huorka M, Koller T, et al. Colorectal cancer screening in patients with ulcerative and Crohn's colitis with use of colonoscopy, chromoendoscopy and confocal endomicroscopy. Eur J Gastroenterol Hepatol 2011;23:680-689.

31. Rutter MD, Saunders BP, Wilkinson KH, Kamm MA, Williams CB, Forbes A. Most dysplasia in ulcerative colitis is visible at colonoscopy. Gastrointest Endosc 2004;60:334-339.

32. Rubin DT, Rothe JA, Hetzel JT, Cohen RD, Hanauer SB. Are dysplasia and colorectal cancer endoscopically visible in patients with ulcerative colitis? Gastrointest Endosc 2007;65:9981004.

33. Blonski W, Kundu R, Lewis J, Aberra F, Osterman M, Lichtenstein GR. Is dysplasia visible during surveillance colonoscopy in patients with ulcerative colitis? Scand J Gastroenterol 2008; 43:698-703.

34. Iacucci M, Kaplan GG, Panaccione R, et al. A randomized trial comparing high definition colonoscopy alone with high defi- 
nition dye spraying and electronic virtual chromoendoscopy for detection of colonic neoplastic lesions during IBD surveillance colonoscopy. Am J Gastroenterol 2018;113:225-234.

35. Freire P, Figueiredo P, Cardoso R, et al. Surveillance in ulcerative colitis: is chromoendoscopy-guided endomicroscopy always better than conventional colonoscopy? A randomized trial. Inflamm Bowel Dis 2014;20:2038-2045.

36. Jaramillo E, Tamura S, Mitomi H. Endoscopic appearance of serrated adenomas in the colon. Endoscopy 2005;37:254-260.

37. Spring KJ, Zhao ZZ, Karamatic R, et al. High prevalence of sessile serrated adenomas with BRAF mutations: a prospective study of patients undergoing colonoscopy. Gastroenterology 2006;131:1400-1407.

38. Hurlstone DP, Sanders DS, Lobo AJ, McAlindon ME, Cross SS. Indigo carmine-assisted high-magnification chromoscopic colonoscopy for the detection and characterisation of intraepithelial neoplasia in ulcerative colitis: a prospective evaluation. Endoscopy 2005;37:1186-1192.

39. Carballal S, Maisterra S, López-Serrano A, et al. Real-life chromoendoscopy for neoplasia detection and characterisation in long-standing IBD. Gut 2018;67:70-78. 